\title{
Myeloproliferative disorders: a paradox of in-vivo and in-vitro platelet function
}

\author{
B. J. BOUGhton, W. E. N. CORBETT, AND A. D. GinsbuRG \\ From the Departments of Medicine and Pathology, Queens University, Kingston, Ontario, Canada
}

SUMMARY A patient with features of a myeloproliferative disorder developed an acute multisystems illness and died. In-vitro platelet aggregation was impaired, but necropsy revealed widespread platelet-rich thromboemboli and multiple organ infarctions. It is suggested that platelets are damaged during disseminated intravascular platelet aggregation (DIPA) and that disaggregation of platelet thrombi and recirculation of platelets give rise to their subsequent hypofunction when tested in vitro.

Haemorrhagic and thrombotic disorders are common in myeloproliferative disorders (MPD) (Wasserman and Gilbert, 1966) in which a wide spectrum of platelet function has been described.

Accelerated in-vitro aggregation has been reported during clinical thromboembolic episodes (Vreeken and Van Aken, 1971; Preston et al., 1974), and platelet inhibition with aspirin is therapeutically useful in these cases.

Impaired in-vitro aggregation is more frequent (Berger et al., 1973), but this pattern is paradoxically associated with thrombosis as well as bleeding. Since the platelet hypofunction appears not to protect against thrombosis, we should like to propose a new concept of the pathogenesis of platelet dysfunction in myeloproliferative disease.

\section{Case report}

A 70-year-old man was admitted to hospital with an acute exacerbation of chronic bronchitis. He gave a history of angina pectoris and intermittent claudication, and a recent episode of transient, left-sided hemiparesis. Physical examination revealed features of chronic obstructive lung disease but no residual central nervous system deficit. The spleen was palpable and he was normotensive.

Laboratory studies showed $\mathrm{Hb} 18.0 \mathrm{~g} / \mathrm{dl}$, haematocrit $58.6 \%$, and WBC count $17.7 \times 10^{9} / 1(89 \%$ polymorphonuclear leucocytes with a left shift but no blasts). RBCs showed normochromia, anisopoikilocytosis, and some tear-drop forms, and the platelet count was $865 \times 10^{9} / 1$ with increased megathrombocytes. The leucocyte alkaline phos-

Received for publication 26 July 1976 phatase score was 307 , the ESR was $1 \mathrm{~mm} /$ hour, and bone marrow showed panhyperplasia of all $\supset$ cell series. Renal function tests were normal, uric $\overrightarrow{0}$ acid was $10 \mathrm{mg} / \mathrm{dl}$, and arterial blood gases showed $\forall$ $\mathrm{PaO}_{2} 68 \mathrm{mmHg}, \mathrm{P}_{\mathrm{a}} \mathrm{CO}_{2} 33 \mathrm{mmHg}, \mathrm{pH} 7.45, \mathrm{SO}_{2}$ $94 \%$, and $\mathrm{HCO}_{3}$ ' $21 \mathrm{mEq} / \mathrm{l}(21 \mathrm{mmol} / \mathrm{l})$. The red cell mass and plasma volume were not measured.

Platelet aggregation studies performed on admission, using standard techniques (Ginsburg, $\mathbb{D}$ 1975), showed marked unresponsiveness to adenosine diphosphate, collagen, and adrenaline (Fig. 1).

On a regimen of penicillin, cloxacillin, theophylline, corticosteroids, and oxygen, blood gases became normal, but on the second day he developed a right-sided hemiparesis with cerebrospinal fluid evidence of subarachnoid haemorrhage. The platelet $\dot{0}$ count fell to $420 \times 10^{9} / 1$ and remained at this level until death. Disseminated intravascular coagulation $\delta$ was suspected, but coagulation screening tests were normal, fibrinogen was $3.0 \mathrm{~g} / \mathrm{l}$, and fibrin degrada- 음

tion products were not raised.
On the tenth day he developed positive faecal occult blood and widespread skin petechiae. $A \stackrel{\bar{N}}{ }$ phlebotomy of $450 \mathrm{ml}$ was performed, but on the twelfth day he sustained a myocardial infarction $N$ and died of a cardiac arrest the next day.

NECROPSY FINDINGS

Atherosclerosis was present in the aortic, coronary, $\frac{1}{\Phi}$ renal, and cerebral arteries. Many aortic plaques $\stackrel{\mathscr{S}}{\leftarrow}$ were covered by fresh polypoid thrombi (Fig. 2) $\square$ consisting of granular platelet material and fibrin ${ }_{\vec{P}}$ (Fig. 3). PTAH staining confirmed the large numbers of platelets in these lesions (Fig. 4). The left coronary artery contained fresh occlusive thrombus and the $\frac{\sigma}{\sigma}$ myocardium showed recent infarction and endo- 

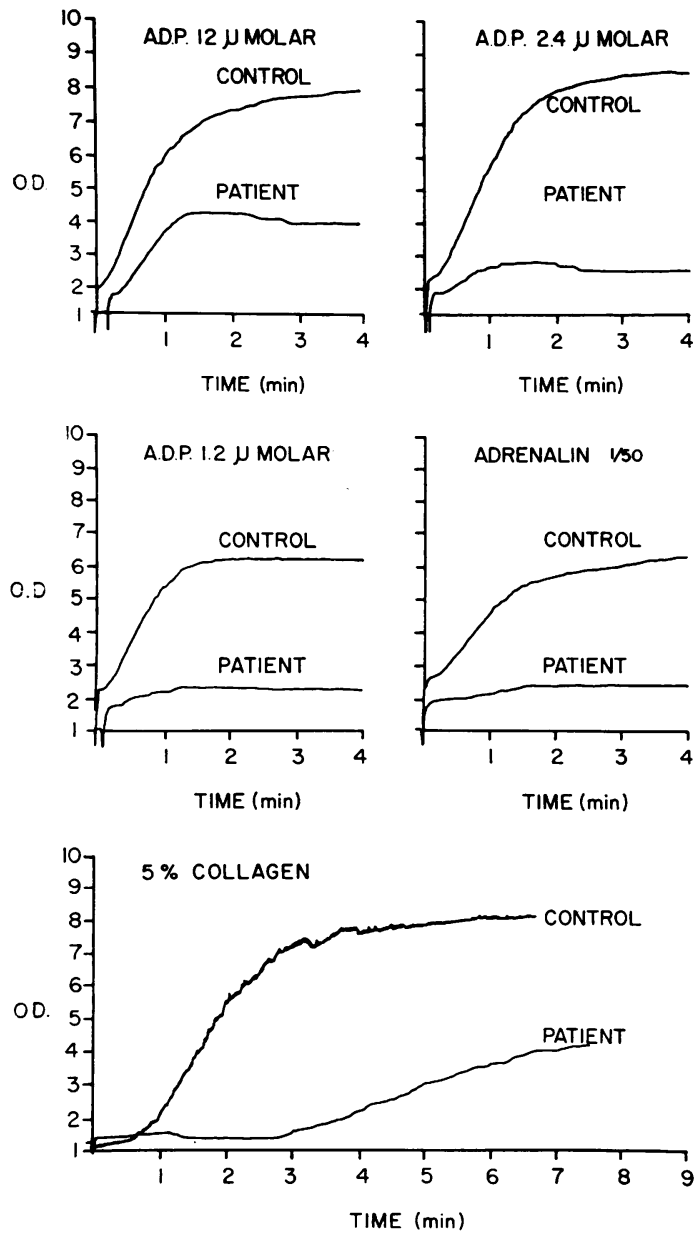

Fig. 1 Platelet aggregation studies in the patient and a normal control.

cardial petechiae. Many intramyocardial arteries contained platelet-rich thrombi (Fig. 5).

Similar thromboemboli were present in the cerebral and renal arteries (Figs. 6 and 7) and in the splenic vessels. Multiple haemorrhagic infarcts were present in the cerebral cortex, thalamus, cerebellum, kidneys, and spleen.

Skin petechiae were widespread, but no source of gastrointestinal bleeding was found. Emphysematous changes were seen in the lungs, but no pulmonary emboli were present.

\section{Discussion}

The features of this case suggest the diagnosis of a myeloproliferative disorder, despite the possibility of secondary hypoxic polycythaemia. The impaired in-vitro platelet aggregation is common in these disorders (Berger et al., 1973) and cannot be explained by the drugs used in his management (Mustard et al., 1972; Cazanave et al., 1973).

There was a marked difference between in-vitro and in-vivo platelet function and a failure of hypofunctional platelets to prevent thrombus formation. Shortened platelet survival was suggested by the falling platelet count and megakaryocytic hyperplasia, and consumption of platelets was confirmed at necropsy in the widespread platelet-rich thrombi and thromboemboli.

Platelet populations are functionally heterogeneous since megathrombocytes are physiologically more active than their smaller counterparts (Karpatkin, 1969). Large platelets are increased in myeloproliferative disorders (Weinfeld et al., 1975) and were elevated in this patient. Though Fig. 1 shows the majority of patient's platelets to be functionally impaired, a small number do appear to be sensitive to the various reagents used. It is, therefore, likely that the platelet-rich thrombi seen at necropsy were generated from the circulating platelet population which had retained some normal function.

Both impaired and accelerated platelet function is reported in myeloproliferative disease (vide supra), and aggregation patterns can change in individual patients. Phlebotomy may improve platelet aggregation in polycythaemia rubra vera (Berger et al., 1973), and we have observed this in $40 \%$ of our patients within three days of phlebotomy. Unlike chemotherapy, phlebotomy would not be expected to reduce the production of abnormal platelets by malignant megakaryocytes, and another explanation is needed to explain these observations. Figure 8 summarises our proposals and is supported by much evidence in the literature.

Ultrastructural studies in myeloproliferative disease have shown normal platelet granulation within the bone marrow (Lagerlöf and Franzén, 1972), in contrast to the marked degranulation of peripheral blood platelets (Maldonado et al., 1974). The delivery of functionally normal platelets to the bloodstream in response to phlebotomy is further evidence that thrombopoiesis is probably normal in these disorders.

Myeloproliferative patients are known to experience episodes of disseminated intravascular platelet aggregation (Vreeken and Van Aken, 1971; Preston et al., 1974) and circulating platelet aggregates have been shown in a number of clinical circumstances (Wu and Hoak, 1974). The microvascular symptoms common to myeloproliferative disease are probably due to small vessel platelet aggregates, and in our experience they respond 


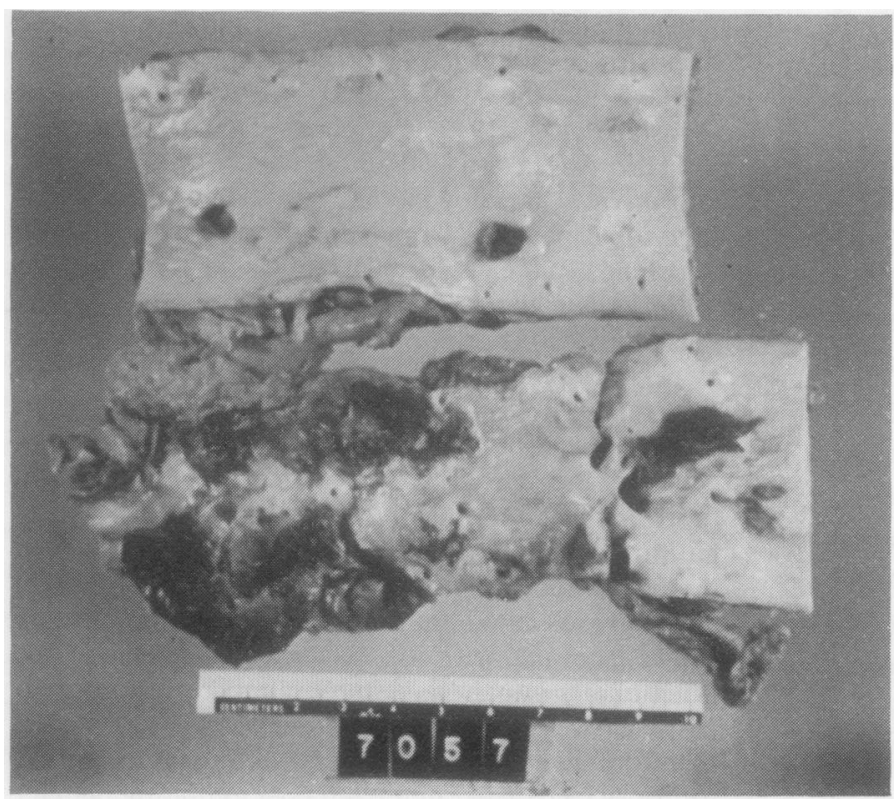

Fig. 2 Portions of thoracic aorta (above) and lower abdominal aorta (below).

Note multiple pale atheromatous plaques and dark polypoid mural thrombi.

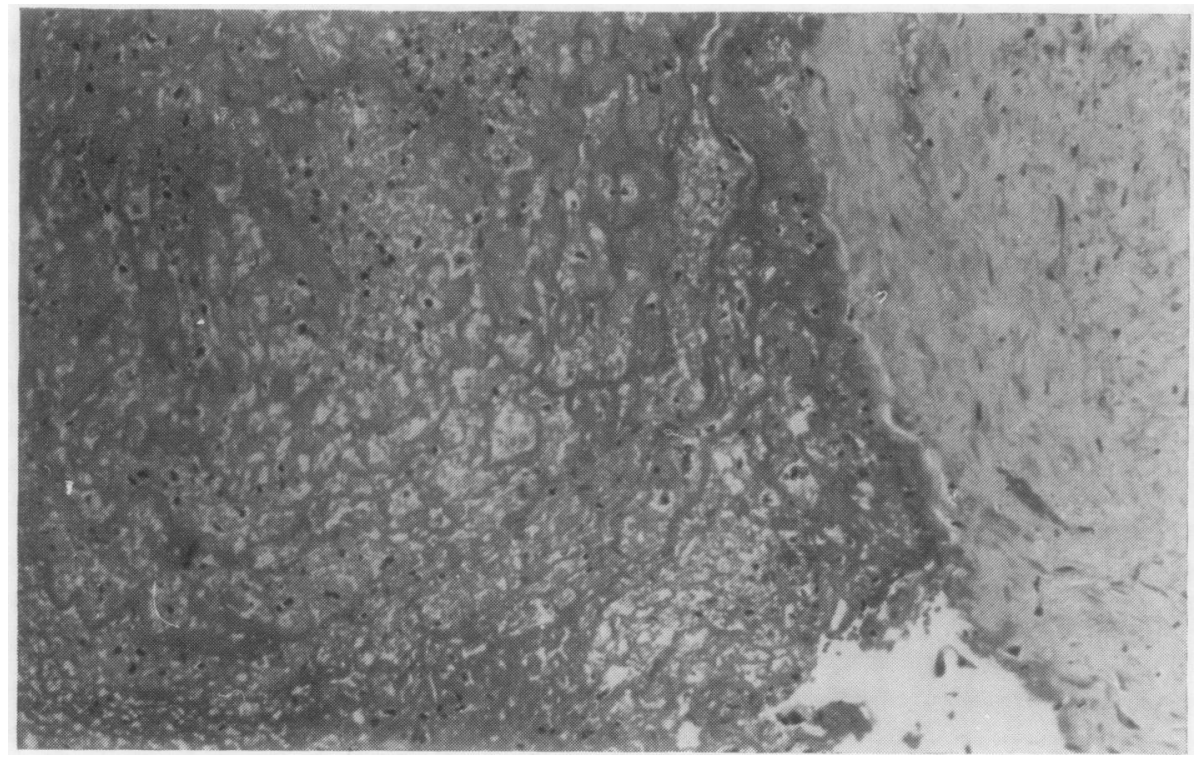

Fig. 3 Aorta. Mural thrombus (left) covering intimal atherosclerotic plaque (right). Thrombus is composed of dark fibrin mesh with intervening pale zones of platelets. (HPS stain $\times 125)$ 


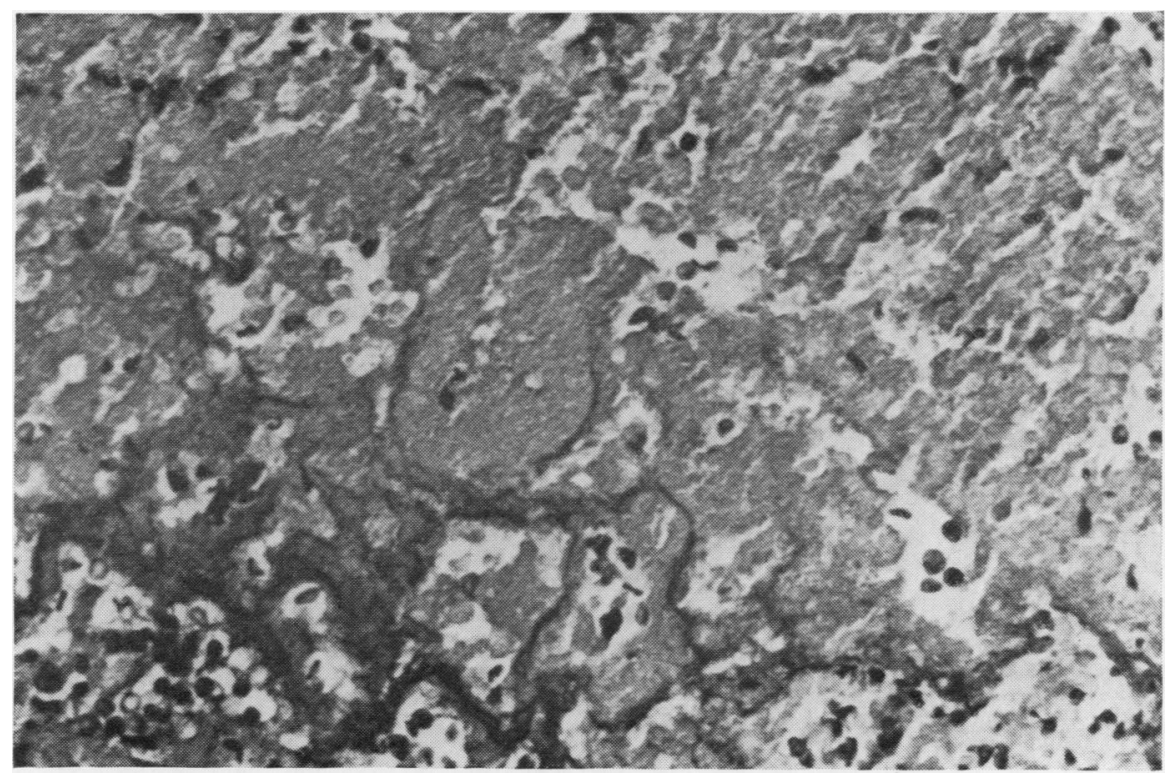

Fig. 4 Detailed structure of thrombus from Figure 3. Note darkly stained PTAH-positive fibrin strands lying between masses of pale, finely granular, aggregated platelets. Also present are scattered pyknotic nuclei of degenerating neutrophils. (PTAH stain $\times 300)$

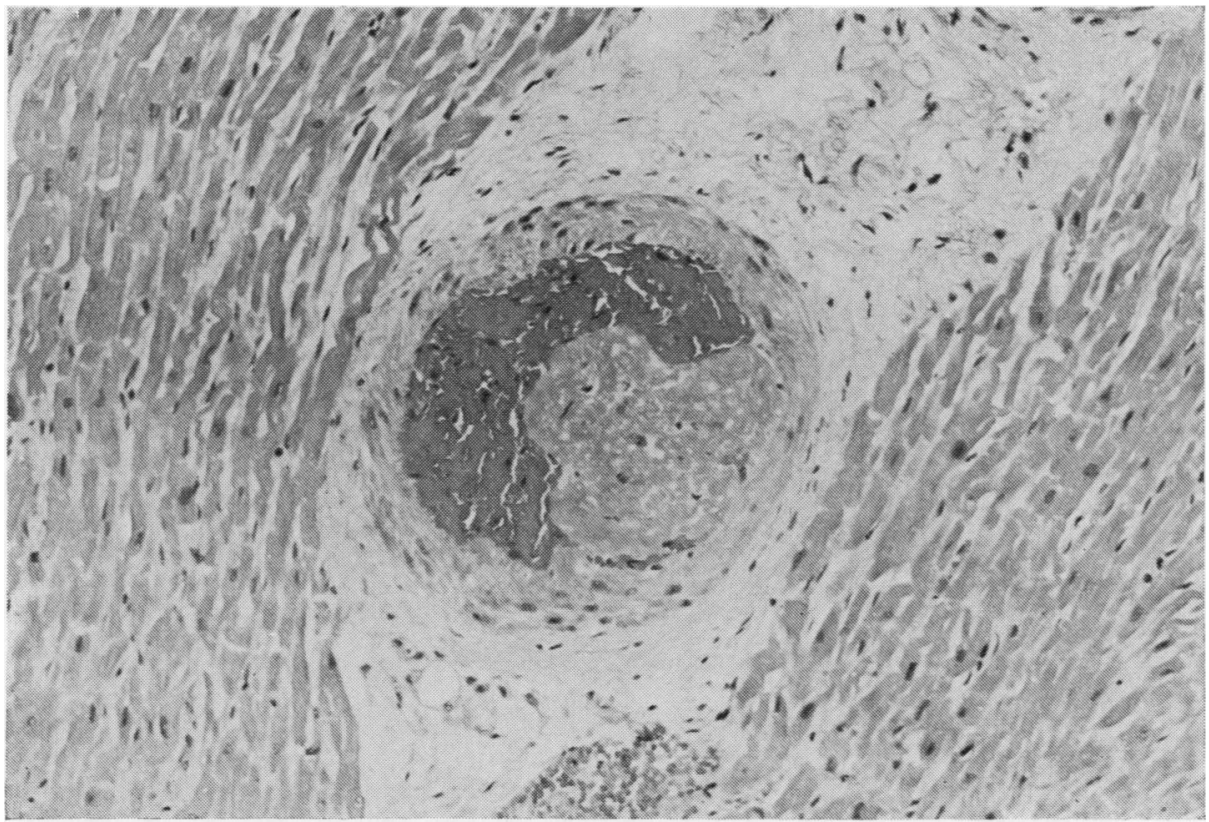

Fig. 5 Small intramyocardial branch of coronary artery. Note platelet aggregate of pale granular material partially occluding lumen. PTAH stains for fibrin were negative. (HPS stain $\times 125)$ 


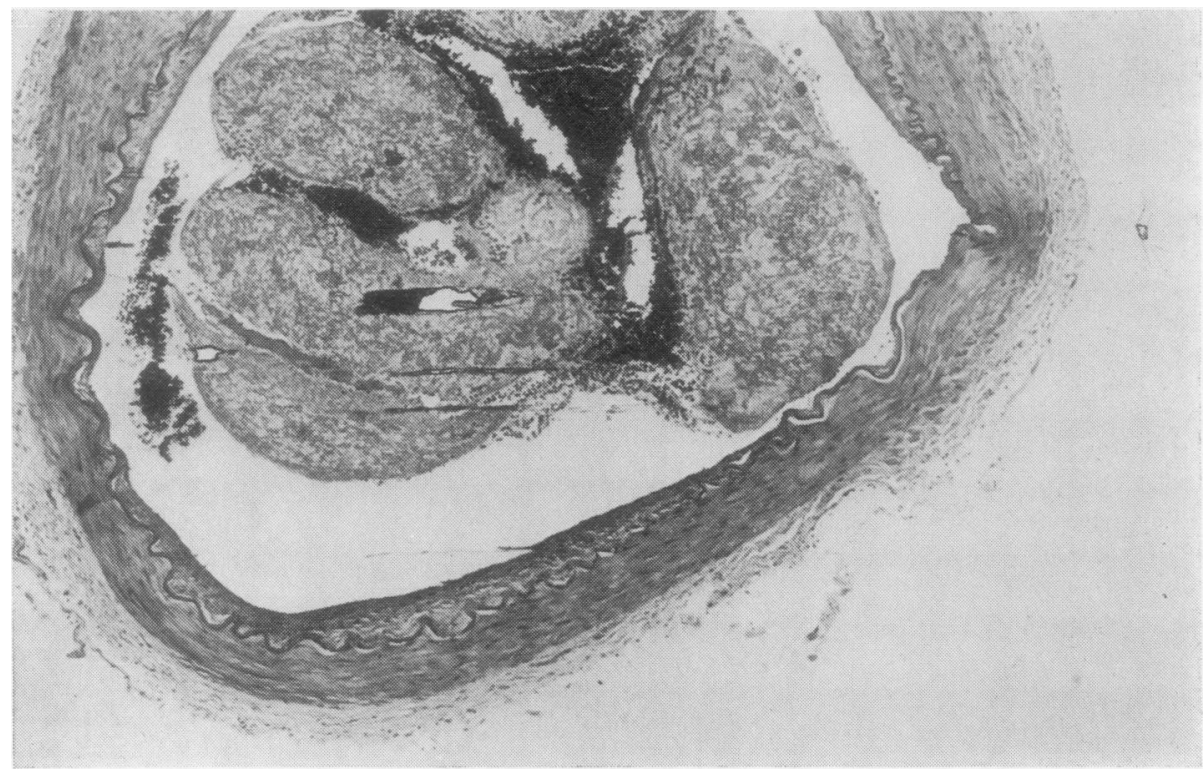

Fig. 6 Anterior cerebral artery containing thromboembolus. $($ HPS stain $\times 125)$

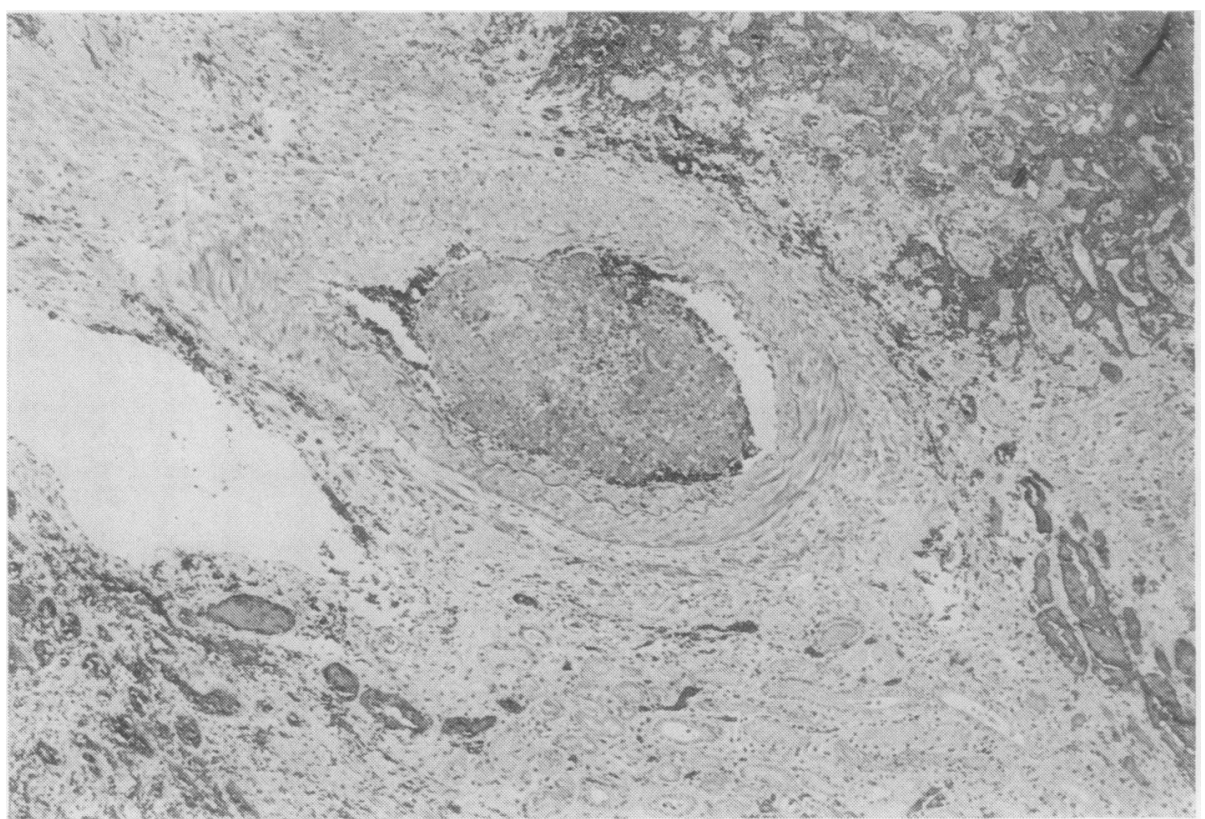

Fig. 7 Interlobar artery of kidney containing thromboembolus. Margin of recent infarct, with haemorrhage, in right upper corner. (HPS stain $\times 125)$ 


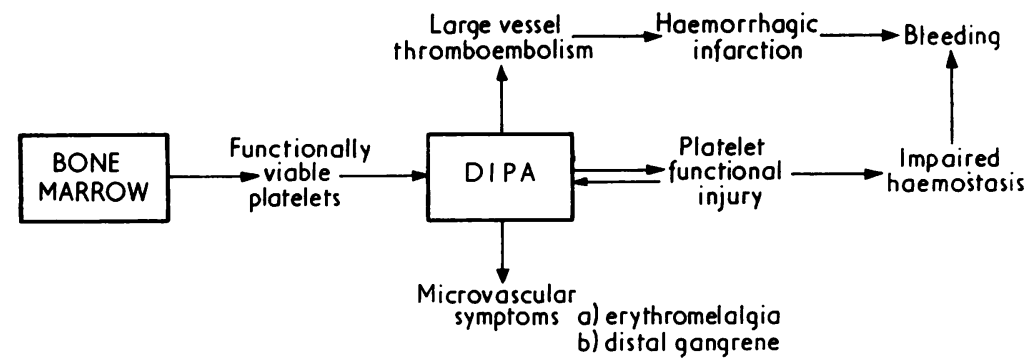

Fig 8 Scheme showing the events surrounding diffuse intravascular platelet aggregation $(D I P A)$, as proposed in the text.

dramatically to aspirin. Large-vessel thromboemboli are also common and, as in this patient, platelets are involved in this process too.

A recent review (Mustard, 1975) emphasises that platelet aggregates tend to break up in their passage through the microcirculation. Platelets can survive such events (Mustard et al., 1966), and their lifespan in vivo may be unaltered by as many as three episodes of thrombin-induced aggregation and disaggregation (Reimers et al., 1973). Platelet function, however, is compromised by agents which trigger the platelet release reaction, and the aggregation patterns produced by pre-exposure to thrombin or shearstress forces (Reimers et al., 1973; Harbury and Schrier, 1974; Brown et al., 1975) are very similar to the ones seen in myeloproliferative disease. Since the myeloproliferative defect is acquired in the peripheral blood, it is probably due to damage sustained during DIPA.

The overall pattern of in-vivo aggregation would, therefore, depend on a balance between the marrow release of normal platelets and their subsequent injury in the circulation.

A predominantly damaged population of platelets would exacerbate bleeding from sites of infarction or produce a generalised bleeding diathesis. It is likely that the process of DIPA would be selflimited by the accumulation of hypofunctional cells. Excessive thrombus formation, or damage to circulating platelets with subsequent splenic sequestration, may exceed the marrow production of new cells and lead, as in this case, to a fall in the platelet count.

Increased marrow production of viable platelets could occur in response to bleeding, infection, or malignancy. The appearance of a predominantly viable population of platelets would account for the correction in platelet function seen after phlebotomy. If platelet production becomes more excessive, the very large numbers of viable platelets could produce the episodes of clinical DIPA which are accompanied by accelerated or spontaneous in-vitro aggregation (Vreeken and Van Aken, 1971; Preston et al., 1974). In these circumstances, chemotherapeutic marrow suppression or inhibition of platelet function with aspirin can produce remission of symptoms (Preston et al., 1974).

Several factors are likely to precipitate DIPA. Atherosclerotic arterial disease is common to these patients, and thromboembolus formation at proximal sites of atheroma is well documented in polycythaemia rubra vera (Barabas et al., 1973). Arterial disease is probably important in localising the site of symptoms in individual patients. Thrombocythaemia and polycythaemia are also factors which may precipitate DIPA since their correction may also improve platelet function (Berger et al., 1973; Zucker and Mielke, 1972; Spaet et al., 1969). Lastly, since platelet function is normal in patients with secondary thrombocytosis (Ginsburg, 1975), there may be intrinsic features of the myeloproliferative platelet which tend to produce DIPA.

The above speculations explain many of the conflicting reports on platelet function in myeloproliferative disease and have important implications concerning the use of platelet inhibitors, such as aspirin, in these disorders.

\section{References}

Barabas, A. P., Offen, D. N., and Meinhard, E. A. (1973). The arterial complications of polycythaemia vera. Brit. J. Surg., 60, 183-187.

Berger, S., Aledort, L. M., Gilbert, H. S., Hanson, J. P., and Wasserman, L. R. (1973). Abnormalities of platelet function in patients with polycythemia vera. Cancer Res., 33, 2683-2687.

Brown, C. H., III, Leverett, L. B., Lewis, C. W., Alfrey, C. P., Jr., and Hellums, J. D. (1975). Morphological biochemical and functional changes in platelets subjected to shear stress. J. Lab. clin. Med., 86, 462-471. 
Cazanave, J. P., Packham, M. A., Guccione, M. A., and Mustard, J. F. (1973). Effects of penicillin G on platelet aggregation, release, and adherence to collagen. Proc. Soc. exp. Biol. (N.Y.), 142, 159-166.

Ginsburg, A. D. (1975). Platelet function in patients with high platelet counts. Ann.intern. Med., 82, 506-511.

Harbury, C. B. and Schrier, S. L. (1974). The effect of a partial release reaction on subsequent platelet function. J. Lab. clin. Med., 83, 877-886.

Karpatkin, S. (1969). Heterogeneity of human platelets. II. Functional evidence suggestive of young and old platelets. J. clin. Invest., 48, 1083-1087.

Lagerlöf, B. and Franzén, S. (1972). The ultrastructure of megakaryocytes in polycythaemia vera and chronic granulocytic leukaemia. Acta path. microbiol. scand., Section A 80, 71-83.

Maldonado, J. E., Pintado, T., and Pierre, R. V. (1974). Dysplastic platelets and circulating megakaryocytes in chronic myeloproliferative diseases. I. The platelets: ultrastructure and peroxidase reaction. Blood, 43, 797809.

Mustard, J. F. (1975). The problem. In Platelets, Drugs and Thrombosis, edited by J. Hirsh et al., p.1. S. Karger, Basle.

Mustard, J. F., Kinlough-Rathbone, R. L., Jenkins, C. S. P., and Packham, M. A. (1972). Modification of platelet function. Ann. N. Y. Acad. Sci., 201, 343-359.

Mustard, J. F., Rowsell, H. C., and Murphy, E. A. (1966). Platelet economy (platelet survival and turnover).
Brit. J. Haemat., 12, 1-24.

Preston, F. E., Emmanuel, I. G., Winfield, D. A., and Malia, R. G. (1974). Essential thrombocythaemia and peripheral gangrene. Brit. med. J., 31, 548-552.

Reimers, H. J., Packham, M. A., Kinlough-Rathbone, R. L., and Mustard, J. F. (1973). Effect of repeated treatment of rabbit platelets with low concentrations $\widehat{\widetilde{D}}$ of thrombin on their function, metabolism and survival. Brit. J. Haemat., 25, 675-689.

Spaet, T. S., Lejnicks, I., Gaynor, E., and Goldstein, $\vec{\circ}$ M. L. (1969). Defective platelets in essential thrombocythemia. Arch. intern. Med., 124, 135-141.

Vreeken, J. and Van Aken, W. G. (1971). Spontaneous aggregation of blood platelets as a cause of idiopath:c응 thrombosis and recurrent painful toes and fingers. Lancet, 2, 1394-1397.

Wasserman, L. R. and Gilbert, H. S. (1966). Complica- $\omega$ tions of polycythaemia vera. Semin. Haemat., 3, N 199-208.

Weinfeld, A., Branehög, I., and Kutti, J. (1975). Platelets윽 in the myeloproliferative disorders. Clinics in Haemato- $\rightarrow$ $\log y, 4,373-392$.

Wu, K. K. and Hoak, J. C. (1974). A new method for the quantitative detection of platelet aggregates in patients $\subseteq$ with arterial insufficiency. Lancet, 2, 924-926.

Zucker, S. and Mielke, C. H. (1972). Classification of thrombocytosis based on platelet function tests. Correlation with hemorrhagic and thrombotic complications. J. Lab. clin. Med., 80, 385-394. 